ENCYCLOPEEDE Encyclopédie berbère

BERBERE

$26 \mid 2004$

26 | Judaïsme - Kabylie

\title{
Juifs au Sahara (Les)
}

\section{J. Oliel}

\section{OpenEdition}

Journals

Édition électronique

URL : http://journals.openedition.org/encyclopedieberbere/1366

DOI : 10.4000/encyclopedieberbere.1366

ISSN : 2262-7197

\section{Éditeur}

Peeters Publishers

\section{Édition imprimée}

Date de publication : 1 mai 2004

Pagination : 3962-3968

ISBN : 2-7449-0452-X

ISSN : 1015-7344

\section{Référence électronique}

J. Oliel, « Juifs au Sahara (Les) », Encyclopédie berbère [En ligne], 26 | 2004, document J16, mis en ligne le 01 juin 2011, consulté le 14 décembre 2020. URL : http://journals.openedition.org/ encyclopedieberbere/1366; DOI : https://doi.org/10.4000/encyclopedieberbere.1366

Ce document a été généré automatiquement le 14 décembre 2020.

(c) Tous droits réservés 


\section{Juifs au Sahara (Les)}

\section{J. Oliel}

1 Les premières incursions juives en Afrique du Nord furent bien antérieures à la conquête arabe, comme peuvent en témoigner certains éléments épigraphiques et objets trouvés dans les ruines de Carthage ; elles ont été suivies de nombreuses autres, celles des Juifs persécutés et chassés par les Babyloniens puis les Grecs ou déportés et vendus par milliers dans les ports d'Afrique du Nord, par les Romains.

2 Après la destruction du second Temple par Titus, en l'an 70, et surtout consécutivement aux guerres de Cyrénaïque (115 et 118), une population juive, relativement importante, s'est dirigée vers l'ouest, a longé l'Atlas saharien, pour finalement se fractionner et se fixer dans les régions du Mzab, du Touat, du Tafilalet, du Dra' et du Sous, rejoignant des populations amies, juives et berbères, installées de longue date.

\section{Les juifs du Touat}

3 Le Touat-Gourara-Tidikelt, province du Sahara aujourd'hui rattachée à l'Algérie, se trouve à un millier de kilomètres au sud de la Méditerranée. Sa population constituée initialement d'Africains - les Haratines ${ }^{* 1}$-, de Berbères zénètes et de Juifs a fait, grâce à son dynamisme, la prospérité d'une région devenue la plaque tournante du commerce transsaharien $\mathrm{du}_{\mathrm{XII}}^{\mathrm{e}}$ au XIV $\mathrm{X}^{\mathrm{e}}$ siècle, au temps où les caravanes sillonnaient le désert pour approvisionner l'Afrique en sel, cotonnades, cuivre et verroterie, et satisfaire les énormes besoins des royaumes musulmans du Maghreb et de l'Espagne en ivoire, plumes d'autruches et surtout, or en poudre [tiber] et esclaves noirs.

4 L'histoire de l'entité juive touatienne n'a été vraiment connue qu'après l'arrivée des Français, en 1900, la découverte de certains documents d'archives et la mise au jour d'une pierre tombale gravée en hébreu², par E.-F. Gautier en 1903 à Ghormali.

5 Les récits des chroniqueurs arabes locaux - collectés par A.G.P. Martin -, notamment celui du Tamentiti, ont permis de faire la lumière sur ce passé :

«Les Indigènes racontent que les ksour de Tamentit furent créés par les juifs l'année de

l'éléphant. C'est ainsi que les Arabes désignent l'année au cours de laquelle eut lieu

l'expédition qu'Abraha, prince éthiopien, entreprit contre La Mecque pour renverser le 
temple de la Kaaba ; Abraha montait un éléphant blanc. »

«[Les] populations arabes trouvèrent dans ce pays une partie de celles qui l'avaient

mis en culture dès le début : c'étaient les Beni Israël $»^{3}$

6 D’après le voyageur Helal ben Messaoud, venu de Mossoul (Irak) au Touat en compagnie d'exilés juifs [qui] «s'est arrêté en l'année 131 [748-749 après J.-C] à Takhfif, que les juifs avaient déjà évacué; il amenait avec lui des commerçants juifs qui s'y installèrent et $y$ résidèrent. Ils y trouvèrent mention sur les tombeaux des Juifs qui avaient abandonné ce pays, que ceux-ci y étaient arrivés en l'année 4429 de la sortie d'Adam [...]. C'était vers l'an 5 après J.-C. que les kuifs en question étaient arrivés à Takhfif».

"Mon hôte, le Mrabet Sidi Youssef, avait appris de son aïeul que ces Juifs avaient été les premiers habitants du Touat et qu'ils y existaient comme nation en 260 [905]. J'ai pu voir moi-même leurs synagogues et leurs boutiques à arcades ${ }^{4}$. "

7 En l'absence de sources d'origine hébraïque, assez discrètes en général - en dehors de quelques documents provenant des autorités rabbiniques d'Alger, datés des $\mathrm{XIV}^{\mathrm{e}}$ et $\mathrm{Xv}^{\mathrm{e}}$ siècles et attestant l'existence de relations entre les communautés du Touat-Gourara et celles du Maghreb (notamment du Mzab, du Dra', de Tlemcen, Alger et Sijilmassa), ces chroniques, qui ne renseignent pas sur les périodes antérieures aux premières arrivées de musulmans au Touat $-\mathrm{x}^{\mathrm{e}}$ siècle -restent précieuses.

8 Néanmoins, malgré quelques incertitudes, certains épisodes d'histoire locale, sont assez bien connus :

- L'arrivée des Zénètes après le v siècle : venus de l'Est ${ }^{5}$, ils auraient introduit dans ces régions le chameau et le palmier qui devaient révolutionner le Sahara avec la création des oasis et le développement considérable des expéditions caravanières.

- Celles, aux siècles suivants, des juifs d'Irak (vi siècle Mossoul), d'Espagne (613 et 694), d'Arabie (675), autant d'immigrants qui ont apporté des techniques utiles au développement économique de la région.

De fait, l'histoire des juifs du Touat ne sera vraiment connue, de la façon la plus probante, qu'au cours $\mathrm{xx}^{\mathrm{e}}$ siècle, grâce à quelques découvertes exceptionnelles :

- La relation d'Antonio Malfante, marchand génois envoyé à Tamentit en 1447 pour tenter de trouver une source d'approvisionnement en or africain indépendante des États maghrébins, nous renseigne sur le système d'échange des marchés de Tamentit, le commerce caravanier transsaharien, les grands centres commerciaux du bassin du Niger...

10 Malfante a pu juger de visu la situation au Touat et se rendre compte du rôle joué par les Maqil (qu'il nomme Philistins), de l'interdépendance des négociants juifs avec leurs associés musulmans.

11 La relation du marchand génois reste le témoignage unique et précieux de la prospérité touatienne et du rôle joué par les juifs, moins d'un demi-siècle avant la fin de leur étonnante aventure, en 1492.

- Une lettre ${ }^{6}$ de 1235 trouvée dans la Guenizah du Caire ${ }^{7}$ fait état d'un commerce caravanier transitant par le Touat, pour échanger l'or africain et le safran, les lingots d'argent... envoyés de Marrakech, contre des perles, des foulards et des tapis d'Orient expédiés de Fustat (Le Caire)...

12 Écrit en caractères hébraïques et en langue arabe, le document est riche de renseignements sur le commerce caravanier (Égypte-Maroc par le Touat), les incidents de parcours, les prix, le rôle des juifs, qui étaient commanditaires, transitaires, correspondants locaux ou simples chameliers... 
- [Mimoun ben Shmouel, ben Braham, ben Kouby...], le texte de la stèle gravée en hébreu en 1390 à Tamentit où elle fut mise au jour en 1988, confirme s'il en était besoin, l'existence, au XIV siècle, de rabbins érudits et de spécialistes en lithographie.

- Un chant d'Ahellil ${ }^{8}$, toujours en usage chez les Berbères zénètes du Gourara, chante la gloire de Salamo (Salomon ?); il pourrait remonter à l'époque du premier Temple de Jérusalem, dont la destruction (en 587 avant J.-C.) entraîna un exode massif des populations juives, dont certaines, probablement, ont pu fuir vers l'Afrique du Nord (et peut-être le Sahara...)

- La cartographie : leur parfaite connaissance du Sahara a permis aux juifs touatiens dont certains avaient leur commerce des deux côtés de la Méditerranée - de renseigner utilement les cartographes majorquins (Abraham Cresques, Mecia de Villadestes, Angelino Dulcert...); dès les XIII ${ }^{\mathrm{e}}$ et XIV ${ }^{\mathrm{e}}$ siècles, ceux-ci ont pu réaliser des cartes et atlas ${ }^{9}$ plus précis et complets que les traditionnels portulans qui ne représentaient que les côtes et les ports du Maghreb, l'intérieur demeurant terra incognita.

13 Ces progrès devaient grandement faciliter les premiers voyages d'Européens vers l'Afrique noire :

- dès 1446, le Portugais João Fernandès a séjourné en Mauritanie chez un hôte connu sous le nom de Ahude (le Juif) Maïmoun.

- en 1447, Malfante, déjà cité, est arrivé au Touat

- en 1455, Ca da Mosto, un Vénitien, choisit la voie maritime pour arriver à Ouadane en Mauritanie et s'approcher du pays de l'or.

- en 1470, le Florentin Benetto Dei aurait atteint Tombouctou...

\section{La montée des périls}

14 Au Touat, depuis assez longtemps déjà, le bel équilibre était menacé par les arrivées continuelles de tribus nombreuses et souvent turbulentes, ce qui devait aggraver les tensions : conflits et affrontements se sont succédé tout au long du XIV , rendant particulièrement inconfortable la situation des Juifs, désormais minoritaires et regroupés dans un quartier de Tamentit, pour échapper aux attaques.

15 Aucun étonnement donc, a priori, lorsque Léon l'Africain* ${ }^{* 10}$ qui a séjourné dans le Gourara, annonce la fin du petit royaume ${ }^{11}$ juif saharien. Les détails de l'événement ne seront connus que plusieurs siècles plus tard :

16 L'aventure des Juifs touatiens a été brutalement interrompue en 1492, par le fanatisme de certains de leurs voisins musulmans conduits par un prédicateur venu de Tlemcen, le cheikh Abdelkrim el-Maghili.

17 Installé à Tamentit en 1479, ce religieux se scandalisait d'y voir des "Juifs arrogants " auxquels n'était pas appliqué, comme dans tout le Maghreb, le statut infamant des dhimmis ${ }^{12}$. Vers 1490 , il saisira le prétexte de la construction (autorisée par le qadi Al Asnouni) d'une nouvelle synagogue à Tamentit ${ }^{13}$, pour consulter les oulémas [docteurs de la loi] de Fès, Tlemcen, Ténès et Tunis. Deux réponses défavorables au projet des infidèles d'élever leur temple dans un pays où triomphe l'islam constituèrent l'encouragement attendu par el-Maghili, qui souleva ses partisans pour les pousser à détruire l'édifice et s'opposer à une éventuelle résistance. Le succès inespéré de l'opération l'incita, le lendemain, à ordonner le massacre, en promettant 7 mitkals d'or (environ $30 \mathrm{~g}$ ) par tête de Juif assassiné. 
18 Ainsi s'est terminée cette prodigieuse épopée des Juifs du Touat, dont les rescapés se sont partagés entre :

- une adhésion à l'islam, pour demeurer au Touat, ce qui ne préservera en rien ces Mohagrin (humiliés) du mépris de leurs voisins ;

- l'exode éperdu à travers le désert, pour tenter de rejoindre soit les communautés du Nord (Mzab, Tafilalet, Dra', Sous...), soit des groupements installés en Afrique noire ou, à la lisière du désert, chez les Touaregs.

\section{Les Juifs du Dra', du Sous}

19 Certains documents et récits - notamment un manuscrit en langue arabe et caractères hébreux daté de $1180^{14}$ et dont il a existé plusieurs exemplaires ou variantes - relatent l'histoire des Juifs du Dra' (Oued Zitoun) ${ }^{15}$.

20 L'ouvrage nous ramène à une époque (bien antérieure à la conquête musulmane) où deux peuples, les Juifs et les Chrétiens ${ }^{16}$, tour à tour rivaux ou associés, se sont disputé cette région.

21 Ces royaumes juifs du Sud marocain (Dra', Sous...), qui ont pu avoir des prolongements en Mauritani $\mathrm{e}^{17}$, ont été anéantis au moment de la poussée almo-ravide ( $\mathrm{xl}^{\mathrm{e}}$ siècle).

22 La diaspora saharienne et soudanaise :

- en Mauritanie, les M'almin (ces forgerons appelés Ihud jusque dans les années 1950) et dont beaucoup semblaient avoir reçu en héritage les techniques et les motifs de décoration traditionnels des Juifs du sud marocain.

- il ne fait guère de doute que, parmi les Touaregs ${ }^{18}$ de nombreux groupes dont les Inaden et, probablement, les Ida Ousahak (Igdalen de l'Aïr), sont issus du Touat.

23 - en 1864, une tribu d'origine juive, les Daga ${ }^{19}$, vivant parmi les Touaregs Iwellemmeden, fut découverte par le rabbin Mardochée.

- près du lac Fati, dans la boucle du fleuve Niger, d'autres Juifs touatiens qui avaient trouvé asile parmi les Beni Israël ${ }^{20}$ subiront une nouvelle persécution en 1496, à l'instigation, encore une fois, d'El Maghili' ${ }^{21}$.

Que reste-t-il de cette extraordinaire épopée?

\section{Au Touat}

25 - Le réseau particulièrement ingénieux de canalisations souterraines, les fogagir (sing. Foggara), idéalement adapté au terrain, au climat et aux besoins en eau des agriculteurs et des éleveurs, a contribué grandement à la réussite économique et à la renommée du Touat.

- Quelques toponymes toujours en usage, dont un d'origine hébraïque, pourrait remonter à la destruction du Temple de Jérusalem: Fenoughil, et d'autres, arabes ou berbères, rappelant la présence juive ancienne: Ksar Lihoud (le fort des Juifs), Rjem Lihoudi (le tombeau du Juif), Theirat Lihoud (le passage des Juifs)...

- Une formule: "le temps des Juifs", dont se servent encore les Musulmans quand ils évoquent - avec quelque nostalgie - l'abondance et la splendeur passées...

- Un reste de tradition juive dans quelques villages du Touat-Gourara (Igosten, Brinken...) où les habitants n'allument pas de feu le samedi. 
Migrations des Juifs au Sahara et en Afrique de l'Ouest après 1492.

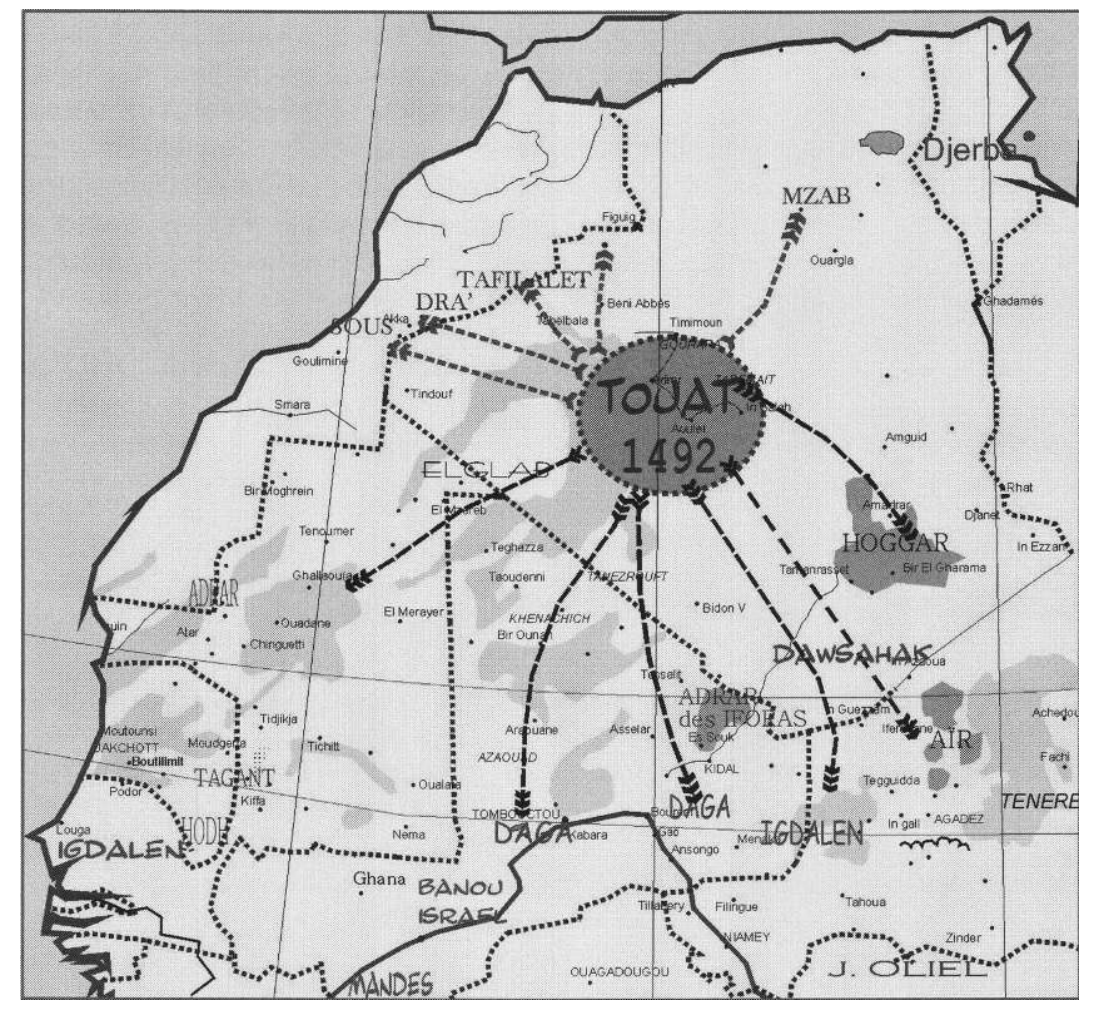

\section{À travers le Sahara}

- Les nombreux descendants des rescapés qui conservent le souvenir de leurs origines juives : Daga, Ida Ousahaq et Igdalen, Inaden...

- Il en a été de même dans l'Adrar de Mauritanie, comme en attestent à la fois les travaux de Colombani, Lucas, Puigaudeau, Richer... et certains éléments qui tiennent à la tradition, l'artisanat ou la toponymie : les bijoutiers-forgerons, appelés Yhud jusque dans les années 1950 et quelques toponymes tels Bir 'Amran et Oudeï Ouled Moussa, dont le nom berbère (littéralement Juifs fils de Moïse) rappelle l'immigration, en 675, des Juifs d'Arabie occidentale, les Beni Moussa [alias Beni Kheïbar, ou Hedjaji] ${ }^{22}$

- Le nom Tabeski, tafaska (dérivé de Pessah) donné par les populations berbérophones à la plus importante fête musulmane. 
D'après Henri Lhote. Les gravures rupestres de l'Atlas saharien..., Alger, Office du Parc National du Tassili, 1984 (291 p.), p. 158 « Station du Rocher des Pigeons ».

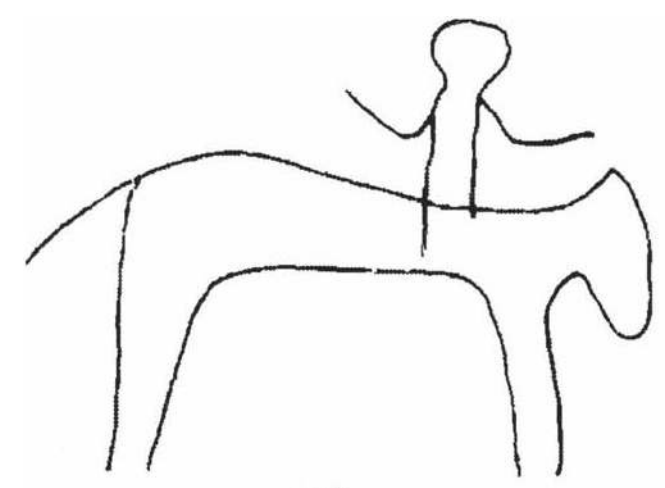

11

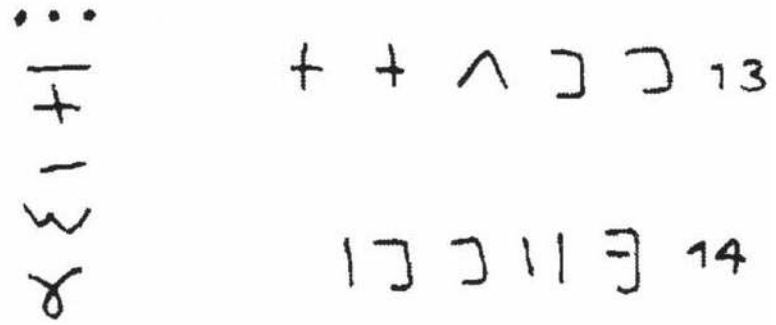

12

Inscription rupestre à caractères Lybico-berbères et hébraïques (lignes 15, 16, 17).

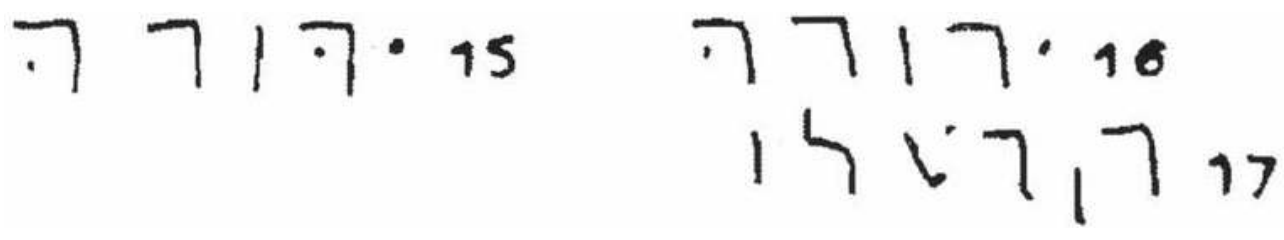

\section{En Europe et ailleurs}

Parmi les descendants de rescapés juifs du Touat,

- Certains portent encore des patronymes rappelant leur origine saharienne : Abani, Gourari, Tamesti, Touati (variantes : Ettouati, Touitou...), Zenati... À l'instar de ceux en usage au Maghreb (Bahloul, Beranès, Medioni, Mimouni, Zeroual...), ils rappellent les liens " préislamiques » entre Berbères et Juifs.

- D'autres continuent, à l'occasion de la fête de Pessah, à émettre le vœu, de se retrouver "L'an prochain à Tamentit », au lieu du classique « l'an prochain à Jérusalem »... 


\section{BIBLIOGRAPHIE}

ET TAMENTITI, « Al Kaoul al bassit fi akhbar Tamentit », in Calassanti Motylinski, Recueil de textes du Touat, B.N.F. ms ar. 6399 (traduction par Louis Watin), Bulletin de la Société de Géographie d'Alger et de l'Afrique du Nord, 1905

JACQUES-MEUNIE D., Le Maroc saharien, des origines à 1670, Klincksiek 1982.

KATI MAHMOUD, Tarîkh el Fettâch (la Chronique du chercheur), traduction O. Houdas et M. Delafosse 1913-1914; réimpression en 1981 in « Documents arabes relatifs à l'histoire du Soudan » (collection UNESCO).

LA RONCIÈRE Ch., Découverte de l'Afrique au Moyen Âge, Le Caire, 1924-1927.

LÉON L'AFRICAIN, Description de l'Afrique, Tierce partie du Monde par Jean-Léon l'Africain, Nouvelle édition annotée par Ch. Scheffer, Paris, 1896.

LÉON L'AFRICAIN, Description de l'Afrique (Nouvelle édition), trad. Epaulard, Paris, 1956.

MARTIN A.G.P., Les Oasis sahariennes, 1908.

MAUNY R., Le Judaïsme, les Juifs et l'Afrique occidentale, Mémoires de l'I.F.A.N. nº 3-4-XI-1949.

MONOD Th., Méharées, 1937.

MONOD Th., L'hippopotame et le philosophe, 1942.

\section{NOTES}

1. Les haratine [sing. Hartani] sont des mélano-gétules d'origine africaine.

2. Celle de Mona, fille de 'Amran, décédée en 1329.

3. Et Tamentiti cité par AGP Martin, les Oasis sahariennes, 1908.

4. Ibid.

5. D'après certains historiens, les Zénètes, déjà présents en Afrique du Nord, au sud de l'Atlas saharien, auraient été bousculés par les Vandales et refoulés vers le désert.

6. Lettre d'Is'haq ben Ibrahim al Touaty provenant de la Genizah du Caire (Cambridge University Library, coll. « Taylor- Schechter », Arabic Box 53, f 66 \& 67).

7. Guénizah: crypte où sont conservés les papiers ou livres renfermant le nom divin, ce qui interdit de les détruire, même s'ils sont devenus inutilisables.

8. Ahellil, mot hébreu de la même racine que halléluïa (glorification).

9. Pour la première fois, furent mentionnés sur les cartes et atlas les noms Touat, Bouda, Tsabit et Tegorarin [le Gourara].

10. Léon, qui le traversa vers 1506, "Tegorarin grande contrée au désert de Numidie. Il y soulait jadis habiter des Juifs fort riches, qui par le conseil et la persuasion d'un prédicateur de Telemsin furent tous saccagés [...], ce qui advint en l'année même que les juifs furent expulsés par Fernand, roi d'Espagne et Sicile».

11. Le mot n'est pas à prendre dans son acception européenne ; le Touat n'ayant ni roi, ni armée, était une entité organisée économiquement.

12. Le statut de Dhimmi imposé aux non-musulmans se traduit par le versement d'un impôt et les marques distinctives de l'abaissement supposant le port d'un costume spécial et d'autres marques d'humiliation... 
13. Synagogues juives du Touat, Archives marocaines II-1908, pp. 244-265.

14. Ms $n^{\circ} 2054$, Fonds Ninar, Institut du Monde Arabe.

15. Le territoire fut, dit la légende, partagé « coudée par coudée ", d'où son nom : DRA'.

16. En 1875, le rabbin Mardochée signalera dans les montagnes de l'extrême Sud marocain un ancien lieu d'implantation de chrétiens (Archives Nationales, 47 A.P. 11 carte : «itinéraire relevé par Mardochée » par Duveyrier).

17. Dès le début, les historiens arabes parlent de Kamnouriyya dont les habitants suivaient la Thora.

18. Théodore Monod (1937, chapitre III, "Bible et Sahara», p. 62), s'était posé, au sujet des Touaregs, cette question : «Hébreux vivants ou Sahariens fossiles?»; simple coïncidence?

19. Alias Daggatoun.

20. Groupement juif de maraîchers connus pour avoir foré des puits dont les parois étaient patinées au beurre de karité, ce qui leur permettait d'obtenir une eau de qualité pour arroser leurs légumes; ils étaient installés dans la région de Tendirma (localité située à $80 \mathrm{~km}$, au sudouest en aval de Tombouctou).

21. Après ses méfaits de 1492 et les réactions de la population musulmane de Tamentit, elMaghili, réfugié à Gao, avait réussi à influencer l'Askia Mohamed Touré, dont le frère Omar Khomzago fit massacrer les Juifs près de Tendirma.

22. D'où, probablement, les patronymes Hedjaji, Hedjazi portés par les Musulmans et, probablement, Hadjej et Hagège, qui ont cours dans les communautés juives de l'est du Maghreb.

\section{INDEX}

Mots-clés : Ahellil, Draa, Epigraphie, Espagne, Forgerons, Niger, Persécutions, Sahara, TouatGourara, Touareg, Synagogue, Sous, Zénètes 